ARTICLE

DOI: $10.1038 /$ s41467-018-05490-0

\title{
Extreme amyloid polymorphism in Staphylococcus aureus virulent PSM $\alpha$ peptides
}

Nir Salinas (D) ${ }^{1}$, Jacques-Philippe Colletier ${ }^{2}$, Asher Moshe ${ }^{1,3} \&$ Meytal Landau (1) ${ }^{1}$

Members of the Staphylococcus aureus phenol-soluble modulin (PSM) peptide family are secreted as functional amyloids that serve diverse roles in pathogenicity and may be present as full-length peptides or as naturally occurring truncations. We recently showed that the activity of PSM $\alpha 3$, the most toxic member, stems from the formation of cross- $\alpha$ fibrils, which are at variance with the cross- $\beta$ fibrils linked with eukaryotic amyloid pathologies. Here, we show that PSM $\alpha 1$ and PSM $\alpha 4$, involved in biofilm structuring, form canonical cross- $\beta$ amyloid fibrils wherein $\beta$-sheets tightly mate through steric zipper interfaces, conferring high stability. Contrastingly, a truncated PSM $\alpha 3$ has antibacterial activity, forms reversible fibrils, and reveals two polymorphic and atypical $\beta$-rich fibril architectures. These architectures are radically different from both the cross- $\alpha$ fibrils formed by full-length PSM $\alpha 3$, and from the canonical cross $-\beta$ fibrils. Our results point to structural plasticity being at the basis of the functional diversity exhibited by S. aureus PSM $\alpha$ s.

\footnotetext{
${ }^{1}$ Department of Biology, Technion-Israel Institute of Technology, Haifa 3200003, Israel. ${ }^{2}$ Institut de Biologie Structurale, Université Grenoble Alpes, Centre National de la Recherche Scientifique (CNRS), Commissariat à l'énergie atomique et aux énergies alternatives (CEA), Grenoble 38044, France. ${ }^{3}$ Present address: School of Molecular Cell Biology \& Biotechnology, George S. Wise Faculty of Life Sciences, Tel Aviv University, Tel-Aviv 6997801, Israel.

Correspondence and requests for materials should be addressed to M.L. (email: mlandau@technion.ac.il)
} 
A myloids designate peptides and proteins capable of selfassembling into structured oligomers and fibrils, and they are mostly known for their involvement in fatal neurodegenerative diseases, such as Alzheimer's and Parkinson's diseases $^{1}$. Some amyloids are functional, in that they participate in specific physiological activities. In humans, functional amyloids partake in immunity, reproduction and hormone secretion ${ }^{2-4}$. In microorganisms, they act as key virulence factors, and may thus represent novel targets for antibacterial drugs ${ }^{5,6}$. For example, amyloid fibrils are found in the self-produced polymeric matrix that embeds biofilm-forming bacteria, where they act as a physical barrier that increases their resilience and resistance to antimicrobial drugs ${ }^{5-8}$ and to the immune system 9 . Functional bacterial amyloids may also act as toxins, killing non-self-cells and thereby increasing virulence $e^{6,7,10}$. The structural hallmarks of functional amyloids-if any-and how they can be distinguished from disease-associated amyloids remain unclear. To date, only a single atomic resolution structure of a functional bacterial amyloid has been made available, namely, that of phenol-soluble modulin a3 (PSMa3), the most cytotoxic member of the Staphylococcus aureus PSMs peptide family. The structure of the fulllength PSM $\alpha 3$ revealed cross- $\alpha$ fibrils ${ }^{10}$, a newly discovered mode of self-assembly characterized by the piling of $\alpha$-helices perpendicular to the fibril axis, in place of $\beta$-strands in canonical cross- $\beta$ amyloid fibrils. Cross- $\alpha$ fibrils ${ }^{10}$ form through the tight mating of $\alpha$-helical sheets, just as cross- $\beta$ fibrils form through the tight mating of $\beta$-sheets ${ }^{11}$; it was thus proposed that they are amyloidlike.

Staphylococcus aureus is a prominent cause of threatening infections ${ }^{12}$ and its PSM family members ${ }^{13}$ serve as key virulence factors that stimulate inflammatory responses, alter the host cell cycle, lyse human cells and contribute to biofilm structuring ${ }^{14,15}$. High expression of PSMas, which are four peptides of about 20residues in length, increases virulence potential of methicillinresistant S. aureus (MRSA) ${ }^{16}$. Amyloid aggregation plays roles in PSM activities in S. aureus. For example, fibrillation of PSM $\alpha 1$ promotes biofilm stability by preventing disassembly by matrixdegrading enzymes and mechanical stress ${ }^{14}$. In addition, fibrillation of PSMa3, enhances its toxicity against human cells ${ }^{10}$. Still, the extent of amyloid fibrillation in all PSM activities remains unclear, especially since PSMs are known to undergo truncation in vivo in response to various external stimuli, yielding truncated PSMs with new functions such as antibacterial activities ${ }^{17-21}$. As each of the truncated PSMs may adopt a different type of amyloid architecture, the array of structural species produced by PSMs is much larger than the actual number of PSMs. In the present study, we investigated the structure-function relationships of PSMas and their short truncations. Our results illuminate the formidable structural plasticity of the multifunctional PSM family.

\section{Results}

The biofilm-associated PSMa1 and PSMa4 form cross- $\beta$ fibrils. We found that in contrast to PSM 33 , which forms cross- $\alpha$ fibrils ${ }^{10}$, the homologous and biofilm-associated ${ }^{14}$ PSMal and PSMa4 form prototypical amyloid fibrils (Fig. 1a, b and Supplementary Fig. 1) which exhibit the cross- $\beta$ signature in X-ray diffraction patterns (Fig. 1c, d), and bind the amyloid-indicator dye thioflavin $\mathrm{T}^{22}$ (Supplementary Fig. 2). It was indeed previously shown that, in solution, PSMa1 and PSMa4 transition from $\alpha$-helices to parallel $\beta$-sheets within several days, whereas PSMa3 remains helical ${ }^{22}$. To obtain atomic-level insights into the architecture of PSMal and PSMa4 fibrils, we computationally identified their amyloidogenic spine segments (Supplementary Fig. 1), and determined their high-resolution structures by means of X-ray microcrystallography (Table 1). Segments PSMa1IIKVIK and PSMa4-IIKIIK, both of which are conserved within naturally occurring PSM truncations ${ }^{18-20}$, adopt the canonical cross- $\beta$ fibril architecture, wherein pairs of $\beta$-sheets tightly mate through a dry interface, forming a steric zipper (Fig. 2, Supplementary Fig. 3a). Both structures belong to the class 1 of steric zippers, indicating that parallel $\beta$-sheets mate face-to-face in the fibrils formed by the PSMa1-IIKVIK and PSMa4-IIKIIK segments ${ }^{23}$. X-ray diffraction from these fibrils accordingly exhibit the cross- $\beta$ pattern (Fig. 2c). Proline substitutions in these spine regions abolished fibril formation of the full-length PSM $\alpha 1$ and PSMa4 (Supplementary Fig. 2). Structural indicators of fibril stability of the IIKVIK and IIKIIK segments, i.e., buried surface area and shape complementarity between sheets, calculated from the crystal structures, resemble those of eukaryotic steric-zipper structures (Supplementary Table 1). The similarity between PSMa1 and PSMa4 segments and human pathological amyloids demonstrate that cross- $\beta$ amyloids are a structural trait shared across species, from bacteria to human.

PSMa3-LFKFFK displays antibacterial activity. PSMa3 is toxic to human cells but certain single-point mutations are known to confer antibacterial activity ${ }^{24,25}$. In addition, natural truncations of PSMs, which occur via proteolysis, could lead to gains in antibacterial activities ${ }^{17-21}$. We found that the LFKFFK segment from PSMa3, identified as fibril-forming by computational methods and which indeed forms fibrils (Fig. 3b), shows dosedependent antibacterial activity against Gram-positive Micrococcus luteus and Staphylococcus hominis, but is nontoxic to S. aureus, the secreting bacterium (Fig. 3a, Supplementary Fig. 4). The observation that the steric-zipper-forming segments PSMa1IIKVIK and PSMa4-IIKVIK do not elicit antibacterial activity (Fig. 3a, Supplementary Fig. 4) indicates that fibrillation is, in itself, insufficient to confer toxicity. Likewise, toxicity of PSM segments cannot be ascribed to charges of their amino acids, since
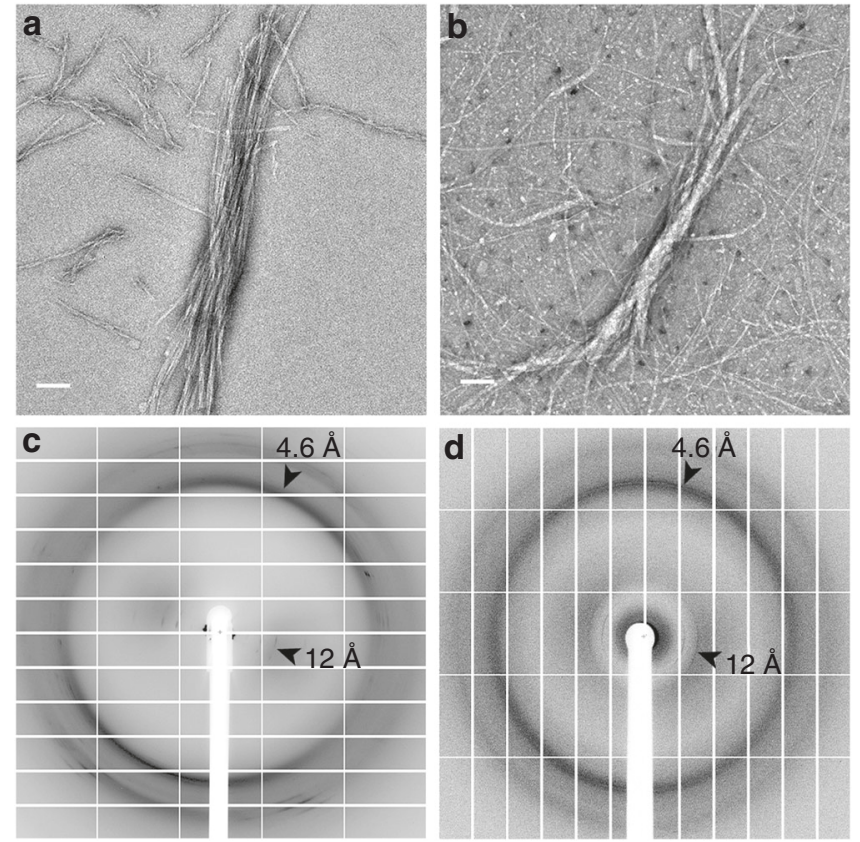

Fig. 1 PSM $\alpha 1$ and PSM $\alpha 4$ form cross- $\beta$ amyloid fibrils. Electron micrographs of PSM 1 1 (a) and PSM $\alpha 4$ (b) show elongated fibrils; scale bars represent $100 \mathrm{~nm}$. X-ray fibril diffraction of PSM $\alpha 1$ (c) and PSM $\alpha 4$ (d) shows major diffraction orthogonal arches at $4.6 \AA$ and $12 \AA$ spacings, indicative of the cross- $\beta$ signature ${ }^{11}$ 
Table 1 Data collection and refinement statistics (molecular replacement)

\begin{tabular}{|c|c|c|c|c|}
\hline & IIKVIK (PSM $\alpha 1)$ & IIKIIK (PSM $\alpha 4)$ & $\begin{array}{l}\text { LFKFFK polymorph I } \\
(\mathrm{PSM} \alpha 3)\end{array}$ & $\begin{array}{l}\text { LFKFFK polymorph } \\
\text { II (PSM } \alpha 3)\end{array}$ \\
\hline PDB accession code & $6 \mathrm{FG} 4$ & $6 \mathrm{FGR}$ & $6 \mathrm{FHC}$ & $6 \mathrm{FHD}$ \\
\hline Beamline & ESRF ID23-2 & ESRF ID23-2 & ESRF ID23-2 & EMBL P14 PETRA III \\
\hline Date & October $8^{\text {th }}, 2014$ & October $8^{\text {th }}, 2014$ & July $24^{\text {th }}, 2014$ & May $2^{\text {nd }}, 2016$ \\
\hline \multicolumn{5}{|l|}{ Data collection } \\
\hline Space group & C 121 & P 1 & $P 65$ & C 121 \\
\hline \multicolumn{5}{|l|}{ Cell dimensions } \\
\hline$a, b, c(\AA)$ & 45.274 .8022 .90 & 4.8322 .3823 .06 & 35.7935 .799 .63 & 41.0311 .7324 .61 \\
\hline$\alpha, \beta, \gamma\left(^{\circ}\right)$ & 90.00107 .6590 .00 & 107.0090 .0196 .20 & 90.0090 .00120 .00 & 90.00121 .8990 .00 \\
\hline Wavelength $(\AA)$ & 0.8729 & 0.8729 & 0.8729 & 0.9763 \\
\hline Resolution $(\AA)$ & $21.8-1.1(1.13-1.10)$ & $22.1-1.5(1.56-1.50)$ & $100-1.5(1.55-1.5)$ & $20.9-1.8(1.96-1.85)$ \\
\hline a R-factor observed (\%) & $13.3(75.2)$ & $20.6(62.2)$ & $8.2(67)$ & $28.4(54.7)$ \\
\hline${ }^{\mathrm{b}} R_{\text {meas }}(\%)$ & $14.0(82.4)$ & $21.7(69.4)$ & $13.1(83.1)$ & $29.2(56.3)$ \\
\hline I / sigma & $10.1(2.1)$ & $7.2(2.4)$ & $20.6(2.3)$ & $9.1(5.6)$ \\
\hline Total reflections & $23,104(876)$ & $13,816(770)$ & 16,288 & $16,102(2590)$ \\
\hline Unique reflections & $2070(136)$ & $1397(158)$ & 1179 (118) & $913(148)$ \\
\hline Completeness (\%) & $93.9(80.0)$ & $95.0(89.3)$ & $97.4(98.3)$ & $96.3(91.9)$ \\
\hline Redundancy & $11.2(6.4)$ & $9.9(4.9)$ & $13.8(9.6)$ & $17.6(17.5)$ \\
\hline${ }^{c} \mathrm{CC}_{1 / 2}(\%)$ & $99.8(96.7)$ & $99.4(74.4)$ & $96.5(97.6)$ & $99.3(94.6)$ \\
\hline \multicolumn{5}{|l|}{ Refinement } \\
\hline Resolution $(\AA)$ & $18.4-1.1(1.23-1.10)$ & $18.2-1.5(1.54-1.50)$ & $31.0-1.5(1.55-1.51)$ & $19.3-1.8(1.90-1.85)$ \\
\hline Completeness (\%) & $94.0(83.5)$ & $95.1(93.9)$ & $97.8(98.9)$ & $97.3(98.8)$ \\
\hline d No. reflections & 1863 & 1257 & 1057 & 821 \\
\hline e $R_{\text {work }}(\%)$ & $15.9(21.2)$ & $18.3(26.4)$ & $11.8(27.7)$ & $17.1(29.7)$ \\
\hline$R_{\text {free }}(\%)$ & $19.4(26.4)$ & $22.1(26.1)$ & $16.2(21.2)$ & $18.7(43.6)$ \\
\hline No. atoms & 77 & 127 & 86 & 136 \\
\hline Protein & 65 & 102 & 60 & 120 \\
\hline Ligand/ion & 10 & 22 & 21 & 11 \\
\hline Water & 2 & 3 & 5 & 5 \\
\hline \multicolumn{5}{|l|}{$B$-factors } \\
\hline \multirow[t]{2}{*}{ Protein } & 8.8 & 7.7 (Chain A) & 10.2 & 9.6 (Chain A) \\
\hline & & 8.2 (Chain B) & & 11.3 (Chain B) \\
\hline \multirow[t]{4}{*}{ Ligand/ion } & $14.5\left(\mathrm{SO}_{4}\right)$ & $37.3\left(\mathrm{SO}_{4}\right)$ & $25.1\left(\mathrm{CO}_{3}\right)$ & $29.1\left(\mathrm{SO}_{4}\right)$ \\
\hline & & $6.7(\mathrm{EDO})$ & $18.3(\mathrm{SCN})$ & $25.9(\mathrm{Na})$ \\
\hline & & & $23.7(P G 4)$ & \\
\hline & & & $46.5(\mathrm{Cl})$ & \\
\hline Water & 33.6 & 26.9 & 49.9 & 23.5 \\
\hline \multicolumn{5}{|l|}{ R.m.s. deviations } \\
\hline Bond lengths $(\AA)$ & 0.010 & 0.010 & 0.016 & 0.017 \\
\hline Bond angles $\left(^{\circ}\right)$ & 1.973 & 1.903 & 1.995 & 1.893 \\
\hline Clash score 75 & 0.00 & 0.00 & 6.1 & 0 \\
\hline Molprobity score 75 & 1.33 & 0.5 & 1.33 & 1.20 \\
\hline Molprobity percentile 75 & 85th percentile & 100th percentile & $94^{\text {th }}$ percentile & $99^{\text {th }}$ percentile \\
\hline $\begin{array}{l}\text { Number of xtals used } \\
\text { for scaling }\end{array}$ & $\begin{array}{l}\text { Four spots from one } \\
\text { crystal were used. }\end{array}$ & $\begin{array}{l}\text { Four spots from one } \\
\text { crystal were used. }\end{array}$ & One crystal & $\begin{array}{l}\text { Three spots from } \\
\text { one crystal were } \\
\text { used. }\end{array}$ \\
\hline \multicolumn{5}{|c|}{$\begin{array}{l}\text { Values in parentheses are for highest-resolution shell } \\
\text { aRfactor square } \\
\text { bR-meas is a redundancy-independent R-factor defined in }{ }^{76} \\
{ }^{{ }^{7} C C_{1 / 2} \text { is percentage of correlation between intensities from random half-datasets }}{ }^{77} \\
\text { dNumber of reflections corresponds to the working set }^{\text {enwork corresponds to working set }}\end{array}$} \\
\hline
\end{tabular}

all three segments feature two basic and four hydrophobic side chains. Thus, the antibacterial activity of the PSMa3-LFKFFK segment could stem from the specific fibril architecture it adopts.

LFKFFK forms reversible fibrils and atypical structures. We obtained two atomic resolution structures of LFKFFK; both revealed a departure from cross- $\beta$ fibrils and atypical amyloid architectures (Fig. 4). One polymorph was fundamentally different from steric-zippers, displaying no dry interface between pairs of $\beta$-sheets. Instead, hexamers of $\beta$-sheets formed cylindrical channels running along the fibril-like structure, effectively yielding nanotubes (Fig. 4a). The second polymorph was composed of out-of-register $\beta$-sheets (Fig. 4b), meaning that unlike in canonical cross- $\beta$ fibrils, $\beta$-strands are not perpendicular to the fibril axis ${ }^{26,27}$. Such extreme polymorphism is exceptional within the hundreds of structures of amyloid-like spine segments solved to date ${ }^{28}$. Quantitative measures of amyloid stability based on the crystal structures $^{28}$ (Supplementary Table 1) suggest that both polymorphs, and especially the hexameric configuration, form less stable fibrils compared to canonical steric zippers. The hexameric configuration lacks the tight interdigitation of $\beta$-sheets characteristic of canonical cross- $\beta$ fibrils, such as PSM $\alpha 4$-IIKVIK and eukaryotic steric-zipper amyloid segments, resulting in a smaller solvent-accessible surface area buried and lower shape complementarity at the interface between the $\beta$-sheets (Supplementary Table 1). The second polymorph, featuring out-ofregister $\beta$-sheets, shows intermediate quantitative measures of 
a

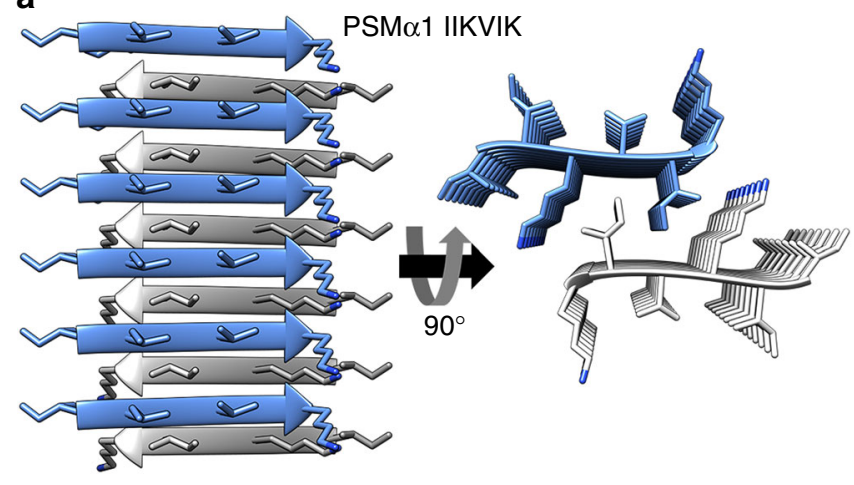

b
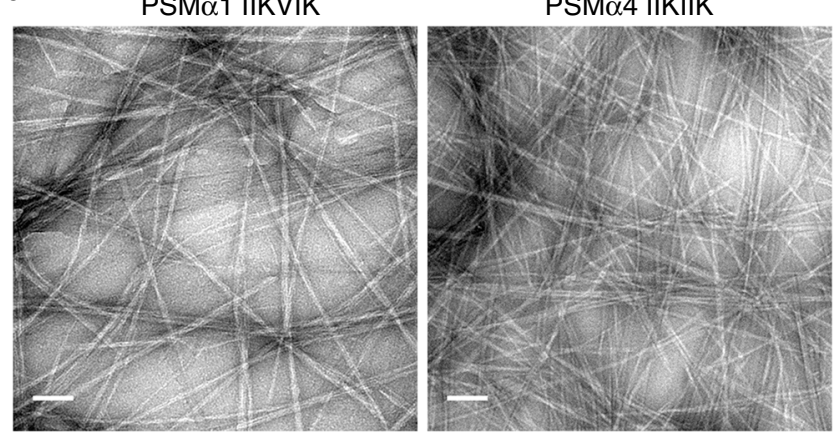

C

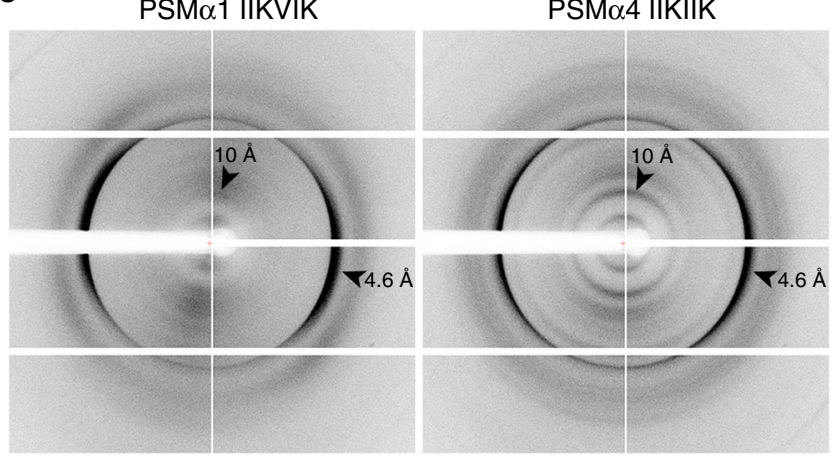

Fig. 2 PSM $\alpha 1$ and PSM $\alpha 4$ spine segments form steric zipper fibrils.

a Crystal structure of the PSM $\alpha 1$ spine segment IIKVIK determined at $1.1 \AA$ resolution, showing the canonical steric-zipper architecture of tightly mated $\beta$-sheets composed of parallel $\beta$-strands. In the left panel, the view is perpendicular to the fibril axis and the $\beta$-strands run horizontally. In the right panel, the view is down the fibril axis. The segments are shown in ribbon representation, with the side chains shown as sticks. The carbons within each $\beta$-sheet are colored either gray or light blue, and nitrogen atoms in side chains are colored blue. Here, six layers of $\beta$-strands are presented. Theoretically, fibrils can contain thousands of layers. A similar structure of the PSM $\alpha 4-I I K I I K$ and its crystal packing are shown in Supplementary Fig. 3. b Electron micrographs visualizing PSM $\alpha 1-I I K V I K$ and PSM $\alpha 4-$ IIKIIK fibrils; scale bars represent $100 \mathrm{~nm}$. c X-ray fibril diffraction of PSM $\alpha 1-$ IIKVIK and PSM $\alpha 4-$ IIKIIK shows major diffraction orthogonal arches at $4.6 \AA$ and $10 \AA$ spacings, indicative of the cross- $\beta$ signature ${ }^{11}$

amyloid stability, consistent with the previous suggestion that this mating configuration could be associated to the transient nature of amyloid oligomers ${ }^{26,29}$. The contrasting levels of stability of PSMa3-LFKFFK and PSMa4-IIKIIK fibrils, inferred from structural analyses, were verified experimentally. Specifically, PSMa3LFKFFK fibrils dissolve upon heating to $50^{\circ} \mathrm{C}$, and reform upon cooling, whereas PSMa4-IIKIIK fibrils remain stable (Fig. 5). This behavior is reminiscent of the reversible fibril formation displayed by low-complexity protein segments associated with membraneless assemblies, which form fibrils with kinked $\beta$-sheets ${ }^{30,31}$, and by the TAR DNA-binding protein 43 (TDP-43) that can aggregate both in reversible stress granules and in irreversible pathogenic amyloid ${ }^{32}$. Similar to these human functional amyloids, the labile fibril formation by PSMa3-LFKFFK could underlie a functional role.

LFKFFK and KLFKFFK share fibril properties and function. The antibacterial activity of LFKFFK is preserved in the oneresidue longer PSMa3 segment KLFKFFK (Fig. 3a, Supplementary Fig. 4). LFKFFK and KLFKFFK both form polymorphic fibrous structures (Fig. 3b), and the secondary structure of their fibrils analyzed using attenuated total-internal reflection Fourier transform infrared (ATR-FTIR) spectroscopy showed similar spectra with the presences of $\beta$-rich species (Supplementary Fig. 5). Specifically, the steric-zipper segment PSMa1-IIKVIK showed a peak at $1621 \mathrm{~cm}^{-1}$ corresponding to rigid cross- $\beta$ amyloid fibrils ${ }^{33-35}$, in accordance with the crystal structure (Fig. 2). Contrastingly, PSMa3-LFKFFK displayed two main peaks at $1622 \mathrm{~cm}^{-1}$ and $1633 \mathrm{~cm}^{-1}$, and PSMa3-KLFKFFK displayed a peak at $1633 \mathrm{~cm}^{-1}$. The latter is typical of bent $\beta$-sheets in protein structures ${ }^{33-35}$ and was associated to disorder within amyloid fibrils ${ }^{33-35}$, in accordance with the atypical and polymorphic $\beta$-rich crystal structures of LFKFFK (Fig. 4). We propose that the antibacterial activities of LFKFFK and KLFKFFK fibrils are encoded in their unique structural properties, including their disordered and polymorphic nature.

\section{Discussion}

Work on human disease-associated amyloids has shown that the vast majority of amyloids are $\beta$-rich and polymorphic by nature $^{28}$, which was suggested to encode different level of neurotoxicity and prion strains ${ }^{36}$. We hypothesize that functional amyloids are even richer in polymorphisms, as they encode highly diverse functions in a reduced number of related sequences. In the present study, we found considerable structural diversity among close homologs (Supplementary Fig. 6). While PSMa3 forms cross- $\alpha$ fibrils and enhances toxicity against human cells ${ }^{10}$, PSMa1 and PSMa4 form cross- $\beta$ fibrils (Fig. 1) which likely play a role in stabilizing the biofilm matrix ${ }^{14}$. Biofilms formed by pathogenic $S$. aureus strains displaying robust amyloid fibril formation indeed contained a high level of PSMa1 and PSMa4 fibrils ${ }^{14,37}$. The role of amyloid in biofilm development is evidenced in other $\beta$-rich microbial amyloids, e.g., curli CsgA from Escherichia coli $^{7}$ and FabC in pseudomonas ${ }^{38}$. The highly stable steric zipper structures (Fig. 2, Supplementary Fig. 3), forming the spines of the cross- $\beta$ fibrils, putatively serve as the building block cementing the biofilm and creating the rigidity that can explain the resistance of amyloid-containing biofilms ${ }^{8,14}$.

A noticeable difference between the steric-zipper structures of the PSMa1 and PSMa4 segments, and the two polymorphs of the LFKFFK segment, is the orientation of the $\beta$-strands, namely parallel vs. antiparallel, respectively (Figs. 2 and 4). Fibrils with $\beta$ sheets arranged in an antiparallel orientation are generally more toxic than those with a parallel arrangement ${ }^{39-42}$. Moreover, the cylindrical architecture of LFKFFK polymorph I (Fig. 4a) is reminiscent of that displayed by a polymorph of amyloid- $\beta$ fibrils ${ }^{43}$, and a HET-s prion fragment ${ }^{44}$. Similarly, stable toxic oligomeric species of $\alpha$-synuclein ${ }^{45}$ and a segment of the human $\alpha \mathrm{B}$-crystalline, named cylindrin ${ }^{29}$ display antiparallel $\beta$-strand orientation and cylindrical architectures. The second polymorph of LFKFFK is composed of out-of-register $\beta$-sheets (Fig. $4 \mathrm{~b}$ ), a morphology that was also suggested to serve as a pathway to toxic 
a

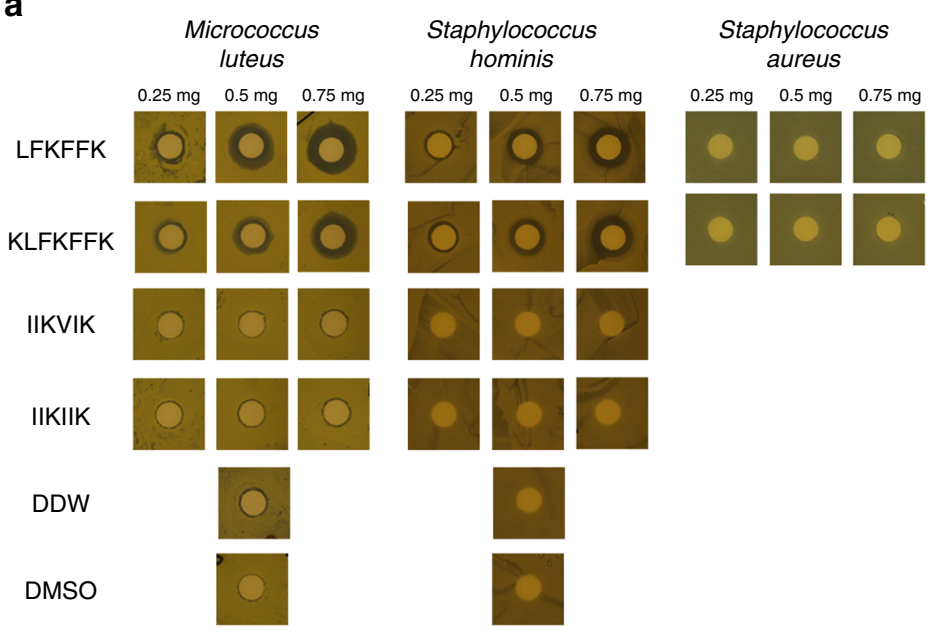

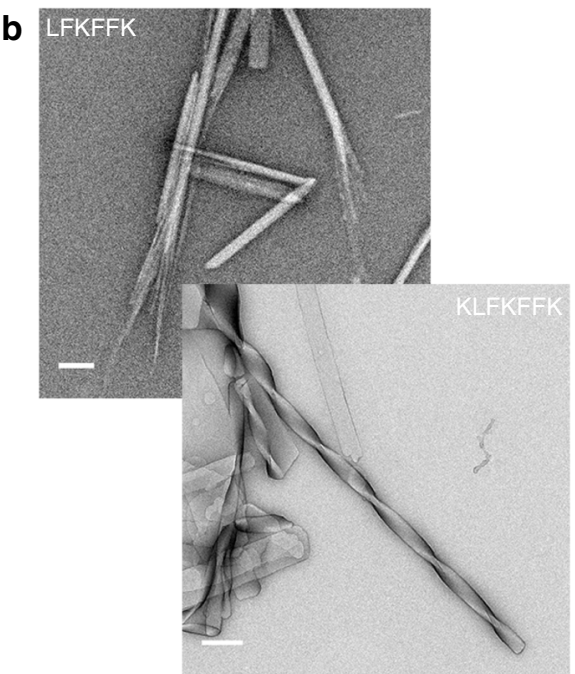

Fig. 3 Antibacterial activity of the fibril-forming LFKFFK from S. aureus PSM $\alpha$ 3. Disc diffusion assay testing antibacterial activity against different bacteria. In this assay, the antibacterial agent diffuses into the agar and inhibits germination and growth of the test microorganism. a LFKFFK and KLFKFFK segments from PSM $\alpha 3$, but not the steric-zipper forming segments PSM 1 1-IIKVIK and PSM $\alpha 4-$ IIKIIK, showed dose-dependent antibacterial activity against M. luteus and S. hominis. LFKFFK and KLFKFFK were not toxic to S. aureus, the bacterium which secretes PSM $\alpha 3$. Discs soaked with only DDW or Dimethyl-sulfoxide (DMSO) served as controls. The results of the disc diffusion assay coincide with the antibacterial activity seen in solution (Supplementary Fig. 4).

b Electron micrographs visualizing fibrils and nano-crystals formed by LFKFFK; scale bar represents $400 \mathrm{~nm}$, and straight and twisted crystalline fibrils of KLFKFFK; scale bar represents $200 \mathrm{~nm}$

a

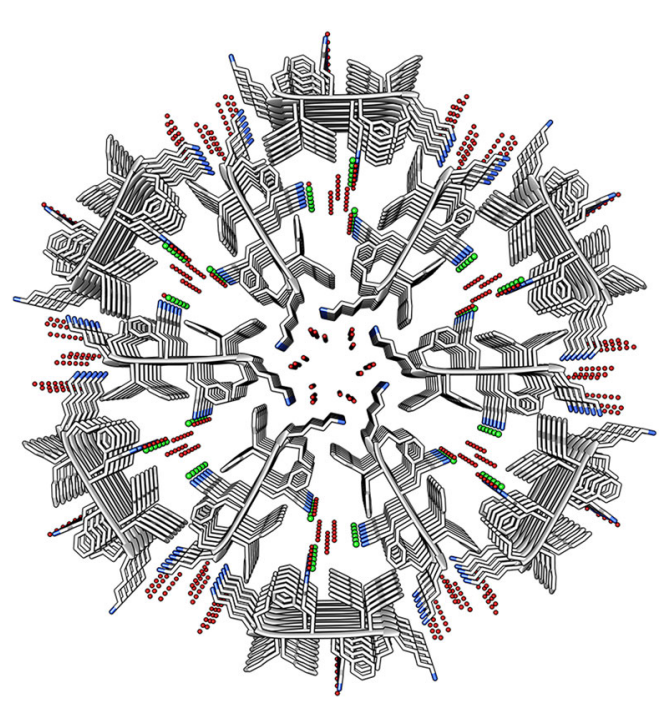

b

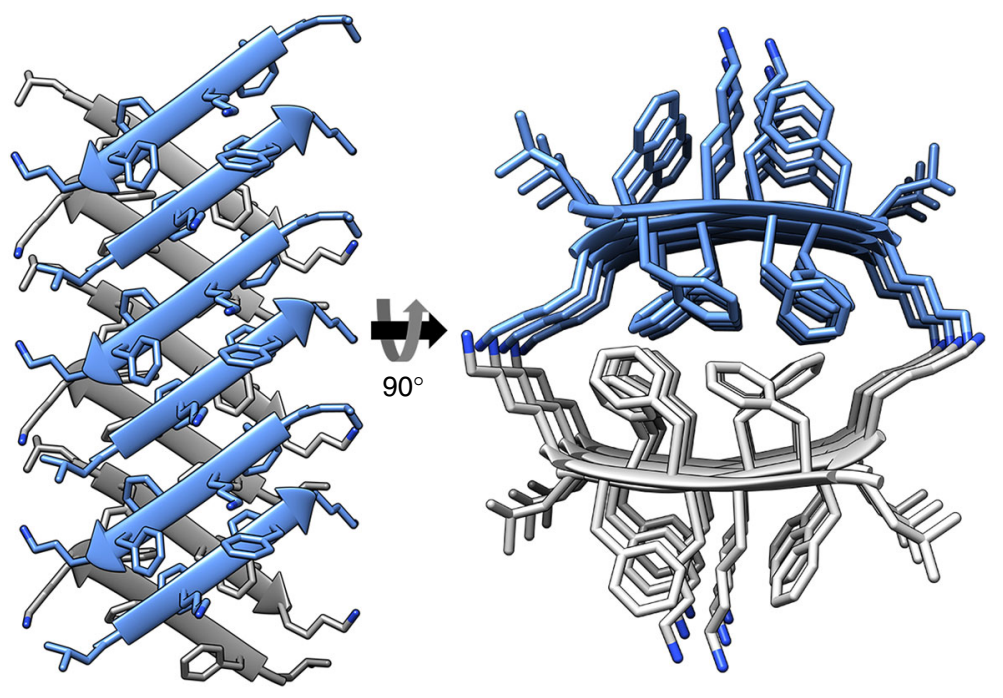

Fig. 4 Structural polymorphism of the LFKFFK segment from S. aureus PSM $\alpha 3$. a Crystal structure of polymorph I of the PSM $\alpha 3$ spine segment LFKFFK determined at $1.5 \AA$ resolution. The structure reveals a unique amyloid-like hexameric architecture, which forms elongated cylindrical cavities along the fibril axis. The view is down the fibril axis. The segments are shown in ribbon representation, with side chains shown as sticks with gray carbons and blue nitrogen atoms. Water molecules (oxygen in red) and chloride ions (green) that counteract the charge of the lysine side chains, are shown as small spheres. $\mathbf{b}$ Crystal structure of LFKFFK polymorph II determined at $1.85 \AA$ resolution, revealed a rare amyloid-like architecture of out-of-register $\beta$-sheets, in which each $\beta$-strand is at an angle of $\sim 50^{\circ}$ from the fibril axis, instead of the close to $90^{\circ}$ angle found for in-register sheets. In both polymorphs, the $\beta$ sheets are composed of anti-parallel strands. In the left panel, the view is perpendicular to the fibril axis, and in the right panel, the view is down the fibril axis. The segments are shown in ribbon representation, with side chain shown as sticks. The carbons within each $\beta$-sheet are colored either gray or light blue, and nitrogen atoms in side chains are colored blue

amyloid aggregates ${ }^{26}$, and the interface between the sheets is reminiscent of the KLVFFA segment of amyloid- $\beta^{42}$. Thus, the two different polymorphs of the antibacterial peptide LFKFFK display features which, in human disease-associated amyloids, correlate with toxicity. Classical steric-zippers and cross- $\beta$ mature fibrils of amyloids are considered to lack the neurotoxicity that has been attributed to smaller, transient, oligomers ${ }^{46}$. This points to transient, less stable and often reversible species with selfassembly properties as the toxic entity in pathological amyloids. We correspondingly suggest that atypical fibrils of LFKFFK encode toxicity to bacteria, while the reversible fibril formation provides means to regulate activity. 


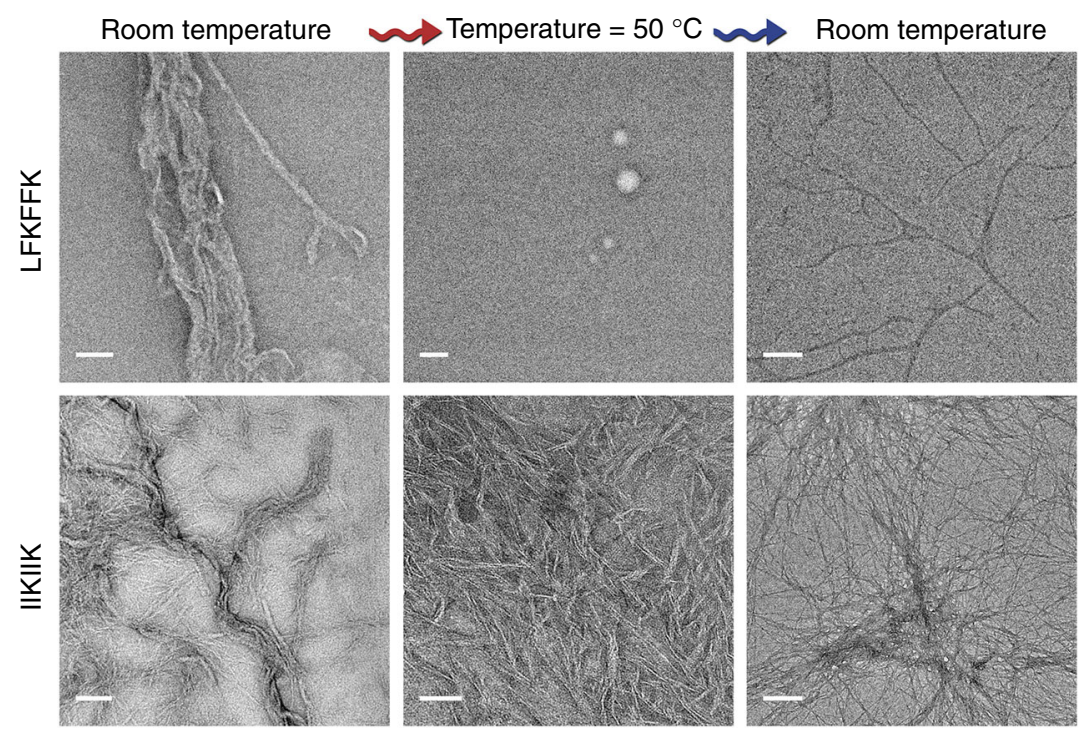

Fig. 5 LFKFFK forms thermo-reversible fibrils compared to the stable IIKIIK fibrils. LFKFFK formed fibrils at room temperature, which dissolved upon heating to $50^{\circ} \mathrm{C}$ and reformed after cooling, albeit with a different morphology; scale bars represent $500 \mathrm{~nm}$ for the sample at room temperature and $300 \mathrm{~nm}$ for the samples taken after heating to $50{ }^{\circ} \mathrm{C}$ and after re-cooling. In contrast, IIKIIK fibrils were thermo-stable; scale bars represent $100 \mathrm{~nm}$ for the sample at room temperature, $50 \mathrm{~nm}$ for the sample taken after heating to $50^{\circ} \mathrm{C}$, and $300 \mathrm{~nm}$ for the sample taken after re-cooling. Both peptides were incubated for 3 days at room temperature before fixation and heating-cooling cycles. The same sample was assayed following temperature change. Thermostability of the fibrils corresponded to the crystal structures; IIKIIK showed the highly stable steric-zipper architecture, while LFKFFK formed atypical amyloid-like structures

The PSMa3 sequence offers a fascinating peek at the complexity of the amyloid fold, populating $\beta$-rich amyloid states in the truncated antibacterial LFKFFK form, but forming cross- $\alpha$ fibril in the context of cytotoxic full-length PSMa $3^{10}$. By not representing a spine that recapitulates the properties of the fulllength PSMa3, the LFKFFK segment exemplifies how an array of activities can be derived from a single polypeptide, through generation of shorter derivatives with different structural properties. This proposal is in agreement with previous observations that PSMs undergo truncations in vivo via proteolysis, yielding derivatives endowed with new functions such as antibacterial activities $^{17-21}$. The biological relevance of the LFKFFK segment is supported by the observation that LFKFFK is nontoxic to the PSMa3-secreting $S$. aureus, but toxic to the closely related $S$. hominis and to M. luteus (Fig. 3). This suggests a mechanism by which $S$. aureus is able to adapt to the toxic fold of LFKFFK fibrils.

A link between amyloids and antibacterial activity has been previously proposed, following the observations that several human host defense peptides form amyloid-like fibrils ${ }^{47,48}$, and that several human pathological amyloids display antimicrobial action $^{49-52}$. LFKFFK presents an additional example. Moreover, LFKFFK associates antibacterial activity with atypical and reversible fibril architectures, which differ from those yielding the highly stable fibrils playing a structural role in biofilms. Remarkably, the shortest recognized peptide that can facilitate antimicrobial activity by molecular self-assembly is diphenylalanine $(\mathrm{FF})^{53}$, which was first identified as the core recognition module of amyloid- $\beta$ involved in Alzheimer's disease ${ }^{54}$. FF forms stiff nanotubes ${ }^{54,55}$, and its crystal structure revealed channels formed via hexagonal symmetry forming elongated tubes ${ }^{56,57}$. The FF motif is present in the LFKFFK segment, and the hexameric structure of LFKFFK resembles the FF nanotubes. This farther support the role of self-assembly in antibacterial activity and specifically the hexamric arrangement that forms channels along the fibril axis.

Together we found that PSMas demonstrate a vast structural diversity of amyloid-like structures, including cross- $\alpha$, cross- $\beta$, out-of-register $\beta$-sheets, and hexameric configurations (Supplementary Fig. 6). By simultaneously secreting PSMas located at the same operon, and by truncations of these peptides, the $S$. aureus bacterium generates various virulent activities encoded by diverse amyloid morphologies. PSMs have critical and diverse roles during infection and represent a promising target for antistaphylococcal therapy ${ }^{15}$. The atomic structures of the PSMa1 and PSMa4 derivatives offer templates for the design of antibiofilm compounds. In addition, the antibacterial activity of LFKFFK and KLFKFFK may be advantageous in the development of antimicrobial peptides based on amyloid segments.

\section{Methods}

Peptides and reagents. PSM native peptides and shorter derivatives (custom synthesis) at $>98 \%$ purity (see Table 2 ) were purchased from GL Biochem (Shanghai) Ltd., as well as from GenScript. The short (6-7 residues) PSMa segments were synthesized with capped termini (acetylated in the $\mathrm{N}$-terminus and amidated in the C-terminus) or with unmodified termini for crystallography. PSMa full-length peptides were synthesized with unmodified termini. Hexafluoroisopropanol (HFIP) and Thioflavin T (ThT) were purchased from SigmaAldrich. Ultra-pure water was purchased from Biological Industries. Dimethylsulfoxide (DMSO) was purchased from Merck.

PSMas wild type (WT) and mutant peptide sequences (Uniprot accession codes are in parenthesis); segments of short derivatives used here are marked in bold; introduced proline substitutions are underlined.

Peptides pre-treatment. Lyophilized synthetic PSMas were dissolved in HFIP to a concentration of $0.5 \mathrm{mg} \mathrm{ml}^{-1}$ followed by a $10 \mathrm{~min}$ sonication in a bath-sonicator at room temperature. The organic solvent was evaporated using a mini rotational vacuum concentrator (Christ, Germany) at $1000 \mathrm{rpm}$ for $2 \mathrm{~h}$ at room temperature. Treated peptides were aliquoted and stored at $-20{ }^{\circ} \mathrm{C}$ prior to use.

Computational prediction of amyloid spine segments. Amyloidogenic propensities of segments from PSMas were predicted using combined information from several computational methods, including ZipperDB ${ }^{58}$, Tango ${ }^{59,60}$, Waltz ${ }^{61}$, and Zyggregator ${ }^{62}$.

Fibril X-ray diffraction. Pre-treated PSMa1 and PSMa4 peptide aliquots were redissolved in DMSO to $20 \mathrm{mM}$ and immediately diluted 2 -fold in ultra-pure water. Short spine segments with capped termini (IIKVIK from PSMa1, IIKIIK from PSMa4) were re-dissolved to $20 \mathrm{mg} \mathrm{ml}^{-1}$ in ultra-pure water. To prepare the sample, $2 \mu \mathrm{l}$ droplets of the peptide solution were placed between two sealed-end 
Table 2 PSMos wild type (WT) and mutant peptide sequences (Uniprot accession codes are in parenthesis)

\section{Peptide name}

PSM $\alpha 1$ (H9BRQ5)

PSM $\alpha 1$ mutant I7P/K9P

PSM $\alpha 3$ (H9BRQ7)

PSM $\alpha 4$ (H9BRQ8)

PSM $\alpha 4$ mutant I8P/I10P

\section{Sequence}

MGIIAGIIKVIKSLIEQFTGK

MGIIAGPIPVIKSLIEQFTGK

MEFVAK̄F KFFKDLLGKFLGNN

MAIVGTIIKIIKAIIDIFAK
MAIVGTIPKPIKAIIDIFAK

Segments of short derivatives used here are marked in bold and introduced proline substitutions are underlined

glass capillaries. PSM $\alpha 1$ and PSMa4 were incubated at $37^{\circ} \mathrm{C}$ for 2 days to allow fibril formation. IIKVIK and IIKIIK were incubated at room temperature until the drop dried completely. X-ray diffraction of PSMal fibrils was collected at the micro-focus beamline P14 operated by EMBL Hamburg at the PETRA III storage ring (DESY, Hamburg, Germany). X-ray diffraction of PSMa4 fibrils was collected at the micro-focus beamline ID23-2 of the European Synchrotron Radiation Facility (ESRF) in Grenoble, France. X-ray diffraction data from fibrils of IIKVIK and IIKIIK was collected at the micro-focus beamline MASSIF-3 (ID30A-3) of ESRF, Grenoble, France.

Thioflavin T fluorescence fibrillation kinetics assay. Thioflavin $\mathrm{T}(\mathrm{ThT})$ is a widely used "gold standard" stain for identifying and exploring formation kinetics of amyloid fibrils, both in vivo and in vitro ${ }^{63}$. Fibrillation curves in presence of ThT commonly show a lag time for the nucleation step, followed by rapid aggregation. Pre-treated PSM 1 1, PSMa1 I7P/K9P, PSMa4, and PSMa4 I8P/I10P peptide aliquots were dissolved in DMSO to $10 \mathrm{mM}$ and immediately diluted to $50 \mu \mathrm{M}$ in Tris buffer pH 7.5 for PSM $\alpha 1$ and PSM $\alpha 1$ I7P/K9P, or in ultra-pure water for PSMa4 and PSMa4 I8P/I10P, containing filtered ThT diluted from stock made in ultrapure water. Final concentrations for each reaction were $50 \mu \mathrm{M}$ peptide (PSMa1, PSMa4, or their mutants) and $200 \mu \mathrm{M}$ ThT. Blank solutions were also prepared for each reaction, containing everything but the peptides. The reaction mixture was carried out in a black 96-well flat-bottom plate (Greiner bio-one) covered with a thermal seal film (EXCEL scientific) and incubated in a plate reader (CLARIOstar BMG LABTECH) at a temperature of $37^{\circ} \mathrm{C}$ with $500 \mathrm{rpm}$ shaking for $85 \mathrm{~s}$ before each reading cycle, and up to 1000 cycles of 6 min each. Measurements were made in triplicates. Fluorescence was measured by excitation at $438 \pm 20 \mathrm{~nm}$ and emission at $490 \pm 20 \mathrm{~nm}$ over a period of about $100 \mathrm{~h}$. All triplicate values were averaged, appropriate blanks were subtracted, and the resulting values were plotted against time. Calculated standard errors of the mean are presented as error bars. The entire experiment was repeated at least three times on different days.

Transmission electron microscopy. Fibrillated PSM $\alpha 1$ and PSM $\alpha 4$ samples were collected following the ThT fibrillation kinetics assay (as described above) by combining the contents of 2-3 wells from the plate. Solutions were centrifuged at $21,000 \times g$ and the supernatant was discarded. The pellet containing the fibrils was re-suspended in $20 \mu$ l of ultra-pure water.

Fibril formation of the short segments, IIKIIK, IIKVIK, LFKFFK and KLFKFFK, was examined for peptides dissolved directly from the powder form. IIKVIK from PSMa1 and IIKIIK from PSMa4 were re-suspended to $20 \mathrm{mM}$ with $75 \%$ DMSO in ultra-pure water and incubated for about 1 week at room temperature (Fig. 2b). LFKFFK and KLFKFFK from PSMa3 were re-suspended in ultra-pure water to $10 \mathrm{mM}$. LFKFFK was incubated for about a month and KLFKFFK was incubated for 14 days at room temperature (Fig. 3b).

Five-microliter samples were applied directly onto copper TEM grids with support films of Formvar/Carbon (Ted Pella), which were charged by glowdischarge (PELCO easiGlow, Ted Pella) immediately before use. Grids were allowed to adhere for $2 \mathrm{~min}$ and negatively stained with $5 \mu \mathrm{l}$ of $2 \%$ uranyl acetate solution. Micrographs were recorded using FEI Tecnai G2 T20 S-Twin transmission electron microscope operated at an accelerating voltage of $200 \mathrm{KeV}$ (located at the Department of Materials Science \& Engineering at the Technion, Israel), FEI Tecnai G2 T12 TEM operated at an accelerating voltage of $120 \mathrm{kV}$ (located at the Russell Berrie Electron Microscopy Center of Soft Matter at the Technion, Israel). Images were recorded digitally by a Gatan US 1000 CCD camera using the DigitalMicrograph ${ }^{\circledR}$ software.

Fibril thermal-stability assay. For the fibril thermal-stability assay (Fig. 5), peptides were dissolved to $10 \mathrm{mM}$ (LFKFFK in ultra-pure water and IIKIIK in DMSO) and incubated at room temperature for 3 days. Samples were applied directly onto copper TEM grids and stained as described above. The samples were then incubated at $50^{\circ} \mathrm{C}$ in a pre-heated thermoblock for $5 \mathrm{~min}$ and immediately applied onto TEM grids. The samples were then allowed to cool down at room temperature for $20 \mathrm{~min}$ and applied onto TEM grids. Micrographs were recorded as described above.

Secondary structure analysis using ATR-FTIR spectroscopy. The short spine segments (IIKVIK from PSMa1, LFKFFK and KLFKFFK from PSMa3) were dissolved to $1 \mathrm{mg} \mathrm{ml}^{-1}$ in $5 \mathrm{mM}$ hydrochloric acid $(\mathrm{HCl})$ and sonicated at a bath sonicator for $5 \mathrm{~min}$ at room temperature. The solutions were frozen in liquid nitrogen and lyophilized over-night to complete dryness. The procedure was repeated three times to completely remove Trifluoroacetic acid (TFA) residues from peptide synthesis, as TFA has a strong FTIR signal. The dry peptide was then dissolved in $\mathrm{D}_{2} \mathrm{O}$ to $20 \mathrm{mg} \mathrm{ml}^{-1}$ for IIKVIK, and $40 \mathrm{mg} \mathrm{ml}^{-1}$ for LFKFFK and KLFKFFK immediately prior to measurements. Five microliters of each peptide solution were spread on the surface of the ATR module (MIRacle Diamond w/ZnSe lens 3-Reflection HATR Plate; Pike Technologies) and let dry under nitrogen gas to purge water vapors. Absorption spectra were recorded on the dry samples using Tensor 27 FTIR spectrometer (Bruker Optics). Measurements were performed in the wavelength range of $1500-1800 \mathrm{~cm}^{-1}$ in $2 \mathrm{~cm}^{-1}$ steps and averaged over 100 scans. Background (ATR module) and blank $\left(\mathrm{D}_{2} \mathrm{O}\right)$ were measured and subtracted from the final spectra. The amide I' region of the spectra $\left(1600-1700 \mathrm{~cm}^{-1}\right)$ is presented (Supplementary Fig. 5).

Crystallizing conditions. Peptides synthesized with free (unmodified) termini were used for crystallization experiments. IIKVIK of PSMal and IIKIIK of PSMa4 were dissolved in $0.5 \%$ acetonitrile in ultra-pure water at $10 \mathrm{mM}$, and LFKFFK of PSMa3 was dissolved in ultra-pure water at $10 \mathrm{mM}$, followed by a $10 \mathrm{~min}$ sonication in a bath-sonicator at room temperature and centrifugation at $17,000 \times g$ at $4{ }^{\circ} \mathrm{C}$ prior to crystallization. Peptide solution drops at $10 \mathrm{nl}$ volume were dispensed by the Mosquito automated liquid dispensing robot (TTP Labtech, UK), to crystallization screening plates. All crystals were grown at $20^{\circ} \mathrm{C}$ via hanging-drop vapor diffusion. The drop was a mixture of $10 \mathrm{mM}$ peptide and reservoir solution as follows: IIKVIK: $0.5 \mathrm{M}$ Lithium sulfate; $15 \%$ polyethylene glycol 8000 . IIKIIK: $0.2 \mathrm{M}$ Ammonium sulfate; $20 \%$ polyethylene glycol 3350 . LFKFFK polymorph I: $0.2 \mathrm{M}$ Potassium thiocyanate, $20 \%$ polyethylene glycol 3350 . LFKFFK polymorph II: $0.1 \mathrm{M}$ Sodium acetate $\mathrm{pH}$ 5.1; $45 \%$ polyethylene glycol 400; $0.09 \mathrm{M}$ Lithium sulfate. Micro-crystals grew after a few days and were mounted on glass needles glued to brass pins ${ }^{64}$. Crystals were kept at room temperature prior to data collection.

Structure determination and refinement. X-ray diffraction data was collected at $100 \mathrm{~K}$. The X-ray diffraction data for IIKVIK, IIKIIK, and LFKFFK polymorph I was collected at the micro-focus beamline ID23-EH2 of the European Synchrotron Radiation Facility (ESRF) in Grenoble, France; wavelength of data collection was $0.8729 \AA$ A. The X-ray diffraction data for LFKFFK polymorph II was collected at the micro-focus beamline P14 operated by EMBL at the PETRAIII storage ring (DESY, Hamburg, Germany); wavelength of data collection was $0.9763 \AA$ A . Data indexation, integration and scaling were performed using XDS/XSCALE ${ }^{65}$ or with DENZO/ SCALEPACK ${ }^{66}$. Molecular replacement solutions for all segments were obtained using the program Phaser ${ }^{67}$ within the CCP4 suite ${ }^{67,68}$. The search models consisted of available structures of geometrically idealized $\beta$-strands. Crystallographic refinements were performed with the program Refmac5 $5^{69}$. Model building was performed with $\operatorname{Coot}^{70}$ and illustrated with Chimera ${ }^{71}$. There were no residues that fell in the disallowed region of the Ramachandran plot. Crystallographic statistics are listed in Table 1.

Calculations of structural properties. The Lawrence and Colman's shape complementarity index ${ }^{72}$ was used to calculate the shape complementarity between pairs of sheets forming the dry interface. Area buried was calculated using AREAIMOL ${ }^{73,74}$ with a probe radius of $1.4 \AA$. Calculations were performed using the CCP4 package ${ }^{68}$. The summation of the differences between the accessible surface areas of one molecule alone and in contact with the other strands on the same sheet or opposite sheets, as indicated in Supplementary Table 1, constitutes the reported area buried.

Bacterial strains and culture media. Micrococcus luteus (an environmental isolate) was a kind gift from Prof. Charles Greenblatt from the Hebrew university in Jerusalem, Israel. An inoculum was grown in Luria-Bertani (LB) medium at $30^{\circ} \mathrm{C}$ with $220 \mathrm{rpm}$ shaking overnight. Staphylococcus hominis (ATTC 27844) and Staphylococcus aureus (ATCC 25923) were purchased from ATCC, USA. An inoculum was grown in brain-heart infusion (BHI) media at $37^{\circ} \mathrm{C}$ with $220 \mathrm{rpm}$ shaking overnight.

Disc diffusion assay. Bacterial cultures were grown overnight as described above and diluted 1000-fold to a fresh media until growth reached to optical density of $\sim 0.4$ measured at $600 \mathrm{~nm}\left(\mathrm{OD}_{600}\right)$. The refreshed cultures were plated on LB-agar or BHI-agar according to the bacterial strain. The antibacterial activity of PSM $\alpha$ segments was examined: Lyophilized peptides were dissolved in ultra-pure water (LFKFFK and KLFKFFK) or DMSO (IIKVIK and IIKIIK) to a final concentration of $50 \mathrm{mg} \mathrm{ml}^{-1}$. The concentrated peptide solutions were loaded on blank antimicrobial susceptibility discs (Oxoid, UK). Discs loaded with ultra-pure water or DMSO were used as controls. The discs were gently placed on bacteria plated agar and incubated over-night at the appropriate temperatures. 
Antibacterial activity in solution. Bacterial cultures were grown overnight as described above and diluted 1000-fold to a fresh media until growth reached to $\mathrm{OD}_{600}$ of $\sim 0.4$. Peptides were dissolved to a concentration of $10 \mathrm{mM}$ (LFKFFK and KLFKFFK in ultra-pure water, IIKVIK and IIKIIK in DMSO) and diluted to $1 \mathrm{mM}$ in growth media (LB for M. luteus and BHI for S. hominis). Two-fold serial dilutions of the tested peptides in LB or BHI media ranging from $250 \mu \mathrm{M}$ to $1 \mu \mathrm{M}$ were performed in a sterile 96-well plate. Final DMSO concentration was fixed to $2.5 \%$ in all samples. Wells containing everything but the peptide served as controls. Bacterial growth was determined by measuring the $\mathrm{OD}_{595}$ during a $24 \mathrm{~h}$ incubation at $30^{\circ} / 37^{\circ} \mathrm{C}$ according to the bacterial strain with $250 \mathrm{rpm}$ shaking in a plate reader (FLUOstar omega or CLARIOstar, BMG LABTECH). The experiment was performed in triplicates. All triplicate values were averaged, appropriate blanks were subtracted, and the resulting values were plotted against peptide concentration. Calculated standard errors of the mean are presented as error bars. The entire experiment was repeated at least three times on different days.

\section{Data availability}

The data that support the findings of this study are available on request from the corresponding author. Coordinates and structure factors for the X-ray crystal structures have been deposited in the protein data bank (PDB) with accession codes 6FG4 (IIKVIK), 6FGR (IIKIIK), 6FHC (LFKFFK polymorph I) and 6FHD (LFKFFK polymorph II).

Received: 12 April 2018 Accepted: 10 July 2018

Published online: 29 August 2018

\section{References}

1. Stefani, M. \& Dobson, C. M. Protein aggregation and aggregate toxicity: new insights into protein folding, misfolding diseases and biological evolution. $J$. Mol. Med. 81, 678-699 (2003).

2. Soragni, A. et al. Toxicity of eosinophil MBP is repressed by intracellular crystallization and promoted by extracellular aggregation. Mol. Cell 57, 1011-1021 (2015).

3. Maji, S. K. et al. Functional amyloids as natural storage of peptide hormones in pituitary secretory granules. Science 325, 328-332 (2009).

4. Hewetson, A. et al. Functional amyloids in reproduction. Biomolecules 7, E46 (2017).

5. Chapman, M. R. et al. Role of Escherichia coli curli operons in directing amyloid fiber formation. Science 295, 851-855 (2002).

6. Blanco, L. P., Evans, M. L., Smith, D. R., Badtke, M. P. \& Chapman, M. R. Diversity, biogenesis and function of microbial amyloids. Trends Microbiol. 20, 66-73 (2012)

7. DePas, W. H. \& Chapman, M. R. Microbial manipulation of the amyloid fold. Res. Microbiol. 163, 592-606 (2012).

8. Schwartz, K. \& Boles, B. R. Microbial amyloids-functions and interactions within the host. Curr. Opin. Microbiol. 16, 93-99 (2013).

9. Gallo, P. M. et al. Amyloid-DNA composites of bacterial biofilms stimulate autoimmunity. Immunity 42, 1171-1184 (2015).

10. Tayeb-Fligelman, E. et al. The cytotoxic Staphylococcus aureus PSMalpha3 reveals a cross-alpha amyloid-like fibril. Science 355, 831-833 (2017).

11. Sunde, M. et al. Common core structure of amyloid fibrils by synchrotron Xray diffraction. J. Mol. Biol. 273, 729-739 (1997).

12. Scherr, T. D., Heim, C. E., Morrison, J. M. \& Kielian, T. Hiding in plain sight: interplay between staphylococcal biofilms and host immunity. Front. Immunol. 5, 37 (2014).

13. Mehlin, C., Headley, C. M. \& Klebanoff, S. J. An inflammatory polypeptide complex from Staphylococcus epidermidis: isolation and characterization. J. Exp. Med. 189, 907-918 (1999).

14. Schwartz, K., Syed, A. K., Stephenson, R. E., Rickard, A. H. \& Boles, B. R. Functional amyloids composed of phenol soluble modulins stabilize Staphylococcus aureus biofilms. PLoS Pathog. 8, e1002744 (2012).

15. Cheung, G. Y., Joo, H. S., Chatterjee, S. S. \& Otto, M. Phenol-soluble modulins-critical determinants of staphylococcal virulence. FEMS Microbiol. Rev. 38, 698-719 (2014).

16. Wang, R. et al. Identification of novel cytolytic peptides as key virulence determinants for community-associated MRSA. Nat. Med. 13, 1510-1514 (2007).

17. Jang, K. S., Park, M., Lee, J. Y. \& Kim, J. S. Mass spectrometric identification of phenol-soluble modulins in the ATCC(R) 43300 standard strain of methicillin-resistant Staphylococcus aureus harboring two distinct phenotypes. Eur. J. Clin. Microbiol. Infect. Dis. 36, 1151-1157 (2017).

18. Gonzalez, D. J. et al. Novel phenol-soluble modulin derivatives in communityassociated methicillin-resistant Staphylococcus aureus identified through imaging mass spectrometry. J. Biol. Chem. 287, 13889-13898 (2012).
19. Gonzalez, D. J. et al. Phenol soluble modulin (PSM) variants of communityassociated methicillin-resistant Staphylococcus aureus (MRSA) captured using mass spectrometry-based molecular networking. Mol. Cell Proteom. 13, 1262-1272 (2014).

20. Deplanche, $M$. et al. Phenol-soluble modulin alpha induces G2/M phase transition delay in eukaryotic HeLa cells. FASEB J. 29, 1950-1959 (2015).

21. Joo, H. S., Cheung, G. Y. \& Otto, M. Antimicrobial activity of communityassociated methicillin-resistant Staphylococcus aureus is caused by phenolsoluble modulin derivatives. J. Biol. Chem. 286, 8933-8940 (2011).

22. Marinelli, P., Pallares, I., Navarro, S. \& Ventura, S. Dissecting the contribution of Staphylococcus aureus alpha-phenol-soluble modulins to biofilm amyloid structure. Sci. Rep. 6, 34552 (2016).

23. Sawaya, M. R. et al. Atomic structures of amyloid cross-beta spines reveal varied steric zippers. Nature 447, 453-457 (2007).

24. Cheung, G. Y. et al. Insight into structure-function relationship in phenol soluble modulins using an alanine screen of the phenol-soluble modulin (PSM) alpha3 peptide. FASEB J. 28, 153-161 (2014).

25. Malishev, R. et al. Reciprocal interactions between membrane bilayers and $S$ aureus PSMalpha3 cross-alpha amyloid fibrils account for species-specific cytotoxicity. J. Mol. Biol. 430, 1431-1441 (2018).

26. Liu, C. et al. Out-of-register beta-sheets suggest a pathway to toxic amyloid aggregates. Proc. Natl Acad. Sci. USA 109, 20913-20918 (2012).

27. Soriaga, A. B., Sangwan, S., Macdonald, R., Sawaya, M. R. \& Eisenberg, D. Crystal structures of IAPP amyloidogenic segments reveal a novel packing motif of out-of-register beta sheets. J. Phys. Chem. B 120, 5810-5816 (2016)

28. Eisenberg, D. S. \& Sawaya, M. R. Structural studies of amyloid proteins at the molecular level. Annu. Rev. Biochem. 86, 69-95 (2017).

29. Laganowsky, A. et al. Atomic view of a toxic amyloid small oligomer. Science 335, 1228-1231 (2012).

30. Luo, F. et al. Atomic structures of FUS LC domain segments reveal bases for reversible amyloid fibril formation. Nat. Struct. Mol. Biol. 25, 341-346 (2018).

31. Hughes, M. P. et al. Atomic structures of low-complexity protein segments reveal kinked beta sheets that assemble networks. Science 359, 698-701 (2018).

32. Guenther, E. L. et al. Atomic structures of TDP-43 LCD segments and insights into reversible or pathogenic aggregation. Nat. Struct. Mol. Biol. 25, 463-471 (2018).

33. Sarroukh, R., Goormaghtigh, E., Ruysschaert, J. M. \& Raussens, V. ATR-FTIR a "rejuvenated" tool to investigate amyloid proteins. Biochim. Biophys. Acta 1828, 2328-2338 (2013).

34. Zandomeneghi, G., Krebs, M. R., McCammon, M. G. \& Fandrich, M. FTIR reveals structural differences between native beta-sheet proteins and amyloid fibrils. Protein Sci. 13, 3314-3321 (2004).

35. Moran, S. D. \& Zanni, M. T. How to get insight into amyloid structure and formation from infrared spectroscopy. J. Phys. Chem. Lett. 5, 1984-1993 (2014).

36. Wiltzius, J. J. W. et al. Molecular mechanisms for protein-encoded inheritance. Nat. Struct. Mol. Biol. 16, 973-U998 (2009).

37. Schwartz, K. et al. The AgrD N-terminal leader peptide of Staphylococcus aureus has cytolytic and amyloidogenic properties. Infect. Immun. 82, 3837-3844 (2014)

38. Dueholm, M. S. et al. Functional amyloid in Pseudomonas. Mol. Microbiol. 77, 1009-1020 (2010)

39. Cerf, E. et al. Antiparallel beta-sheet: a signature structure of the oligomeric amyloid beta-peptide. Biochem. J. 421, 415-423 (2009).

40. Celej, M. S. et al. Toxic prefibrillar alpha-synuclein amyloid oligomers adopt a distinctive antiparallel beta-sheet structure. Biochem. J. 443, 719-726 (2012).

41. Berthelot, K., Ta, H. P., Gean, J., Lecomte, S. \& Cullin, C. In vivo and in vitro analyses of toxic mutants of HET-s: FTIR antiparallel signature correlates with amyloid toxicity. J. Mol. Biol. 412, 137-152 (2011).

42. Colletier, J.-P. et al. Molecular basis for amyloid-beta polymorphism. Proc. Natl Acad. Sci. USA 108, 16938-16943 (2011).

43. Paravastu, A. K., Leapman, R. D., Yau, W. M. \& Tycko, R. Molecular structural basis for polymorphism in Alzheimer's $\beta$-amyloid fibrils. Proc. Natl Acad. Sci. USA 105, 18349-18354 (2008).

44. Wasmer, C. et al. Amyloid fibrils of the HET-s(218-289) prion form a beta solenoid with a triangular hydrophobic core. Science 319, 1523-1526 (2008).

45. Chen, S. W. et al. Structural characterization of toxic oligomers that are kinetically trapped during alpha-synuclein fibril formation. Proc. Natl Acad. Sci. USA 112, E1994-E2003 (2015).

46. Ashe, K. H. \& Zahs, K. R. Probing the biology of Alzheimer's disease in mice. Neuron 66, 631-645 (2010).

47. Kagan, B. L. et al. Antimicrobial properties of amyloid peptides. Mol. Pharm. 9, 708-717 (2012)

48. Landreh, M., Johansson, J. \& Jornvall, H. Separate molecular determinants in amyloidogenic and antimicrobial peptides. J. Mol. Biol. 426, 2159-2166 (2014).

49. Soscia, S. J. et al. The Alzheimer's disease-associated amyloid beta-protein is an antimicrobial peptide. PLoS ONE 5, e9505 (2010). 
50. Kumar, D. K. et al. Amyloid-beta peptide protects against microbial infection in mouse and worm models of Alzheimer's disease. Sci. Transl. Med. 8, 340ra372 (2016).

51. Kobayashi, N., Masuda, J., Kudoh, J., Shimizu, N. \& Yoshida, T. Binding sites on tau proteins as components for antimicrobial peptides. Biocontrol. Sci. 13, 49-56 (2008).

52. Beatman, E. L. et al. Alpha-synuclein expression restricts RNA viral infections in the brain. J. Virol. 90, 2767-2782 (2015).

53. Schnaider, L. et al. Self-assembling dipeptide antibacterial nanostructures with membrane disrupting activity. Nat. Commun. 8, 1365 (2017).

54. Reches, M. \& Gazit, E. Casting metal nanowires within discrete self-assembled peptide nanotubes. Science 300, 625-627 (2003).

55. Kol, N. et al. Self-assembled peptide nanotubes are uniquely rigid bioinspired supramolecular structures. Nano. Lett. 5, 1343-1346 (2005).

56. Gorbitz, C. H. Nanotube formation by hydrophobic dipeptides. Chemistry 7 , 5153-5159 (2001).

57. Gorbitz, C. H. The structure of nanotubes formed by diphenylalanine, the core recognition motif of Alzheimer's beta-amyloid polypeptide. Chem. Commun. 14, 2332-2334 (2006).

58. Goldschmidt, L., Teng, P. K., Riek, R. \& Eisenberg, D. Identifying the amylome, proteins capable of forming amyloid-like fibrils. Proc. Natl Acad. Sci. USA 107, 3487-3492 (2010).

59. Fernandez-Escamilla, A. M., Rousseau, F., Schymkowitz, J. \& Serrano, L. Prediction of sequence-dependent and mutational effects on the aggregation of peptides and proteins. Nat. Biotechnol. 22, 1302-1306 (2004).

60. Rousseau, F., Schymkowitz, J. \& Serrano, L. Protein aggregation and amyloidosis: confusion of the kinds? Curr. Opin. Struc. Biol. 16, 118-126 (2006).

61. Maurer-Stroh, S. et al. Exploring the sequence determinants of amyloid structure using position-specific scoring matrices. Nat. Methods 7, 237-242 (2010).

62. Tartaglia, G. G. et al. Prediction of aggregation-prone regions in structured proteins. J. Mol. Biol. 380, 425-436 (2008).

63. Rogers, D. R. Screening for the amyloid with the thioflavin-T fluorescent method. Am. J. Clin. Pathol. 44, 59-61 (1965).

64. Tayeb-Fligelman, E. \& Landau, M. X-ray structural study of amyloid-like fibrils of tau peptides bound to small-molecule ligands. Methods Mol. Biol. 1523, 89-100 (2017).

65. Kabsch, W. X. D. S. Acta Crystallogr. D Biol. Crystallogr. 66, 125-132 (2010).

66. Otwinowski, Z. \& Minor, W. in Processing of X-ray Diffraction Data Collected in Oscillation Mode Vol. 276: Macromolecular Crystallography, Part A (C. W. Carter, Jr. \& R. M. Sweet, eds.) 307-326 (Academic Press, 1997).

67. McCoy, A. J. et al. Phaser crystallographic software. J. Appl. Cryst. 40, 658-674 (2007).

68. Winn, M. D. et al. Overview of the CCP4 suite and current developments. Acta Crystallogr. D Biol. Crystallogr. 67, 235-242 (2011).

69. Murshudov, G. N., Vagin, A. A. \& Dodson, E. J. Refinement of macromolecular structures by the maximum-likelihood method. Acta Crystallogr. D Biol. Crystallogr. 53, 240-255 (1997).

70. Emsley, P., Lohkamp, B., Scott, W. G. \& Cowtan, K. Features and development of Coot. Acta Crystallogr. D Biol. Crystallogr. 66, 486-501 (2010).

71. Pettersen, E. F. et al. UCSF Chimera-a visualization system for exploratory research and analysis. J. Comput. Chem. 25, 1605-1612 (2004).

72. Lawrence, M. C. \& Colman, P. M. Shape complementarity at protein/protein interfaces. J. Mol. Biol. 234, 946-950 (1993).

73. Lee, B. \& Richards, F. M. The interpretation of protein structures: estimation of static accessibility. J. Mol. Biol. 55, 379-400 (1971).

74. Saff, E. B. \& Kuijlaars, A. B. J. Distributing many points on a sphere. Math. Intell. 19, 5-11 (1997).

75. Chen, V. B. et al. MolProbity: all-atom structure validation for macromolecular crystallography. Acta Crystallogr. D. Biol. Crystallogr. 66, 12-21 (2010).

76. Diederichs, K. \& Karplus, P. A. Improved R-factors for diffraction data analysis in macromolecular crystallography. Nat. Struct. Biol. 4, 269-275 (1997).
77. Karplus, P. A. \& Diederichs, K. Linking crystallographic model and data quality. Science 336, 1030-1033 (2012).

\section{Acknowledgements}

We are grateful to M. Sawaya, N. Ben-Tal, M. Chapman, N. Jain, M. Evans, A. Syed, Z. Hayouka, H. Bochnik-Tamir, O. Tabachnikov, T. Gera, E. Tamar, I. Yelin, O. Snitser, and R. Edrei for help with experiments and fruitful discussions. We acknowledge Y. Pazy-Benhar and D. Hiya at the Technion Center for Structural Biology (TCSB), and the Electron Microscopy Center located at the Technion's Department of Materials Science \& Engineering, and the Russell Berrie Electron Microscopy Center of Soft Matter. This research was supported by the I-CORE Program of the Planning and Budgeting Committee and The Israel Science Foundation, Center of Excellence in Integrated Structural Cell Biology (grant no. 1775/12), DFG: Deutsch-IsraelischeProjektkooperation (DIP) (Grant No. LA 3655/1-1), Israel Science Foundation (grant no. 560/16), University of Michigan-Israel Collaborative Research Grant, and BioStruct-X, funded by FP7. J.-P. C. acknowledges financial support by CEA, CNRS, Université Grenoble Alpes, and the Agence Nationale de la Recherche (Grant No. ANR-15-CE18-0005-02). The synchrotron MX data collection experiments were performed at beamlines ID23-EH2 and MASSIF-3 (ID30A-3) at the European Synchrotron Radiation Facility (ESRF), Grenoble, France, and at beamline P14, operated by EMBL Hamburg at the PETRA III storage ring (DESY, Hamburg, Germany). We are grateful to the teams at ESRF and EMBL Hamburg.

\section{Author contributions}

N.S. and M.L. conceived the study and designed the experiments. All authors collected X-ray data. A.M. initiated experiments. N.S. performed the experiments. N.S. and M.L. solved the crystal structures with contributions from J-P.C. N.S., J-P.C. and M.L. wrote the paper.

\section{Additional information}

Supplementary Information accompanies this paper at https://doi.org/10.1038/s41467018-05490-0.

Competing interests: The authors declare no competing interests.

Reprints and permission information is available online at http://npg.nature.com/ reprintsandpermissions/

Publisher's note: Springer Nature remains neutral with regard to jurisdictional claims in published maps and institutional affiliations.

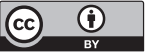

Open Access This article is licensed under a Creative Commons Attribution 4.0 International License, which permits use, sharing, adaptation, distribution and reproduction in any medium or format, as long as you give appropriate credit to the original author(s) and the source, provide a link to the Creative Commons license, and indicate if changes were made. The images or other third party material in this article are included in the article's Creative Commons license, unless indicated otherwise in a credit line to the material. If material is not included in the article's Creative Commons license and your intended use is not permitted by statutory regulation or exceeds the permitted use, you will need to obtain permission directly from the copyright holder. To view a copy of this license, visit http://creativecommons.org/ licenses/by/4.0/

(C) The Author(s) 2018 